\title{
A Magnetic Resonance Imaging-Compatible Device to Perform In Vivo Anterior Knee Joint Loading
}

\author{
Kyoungyoun Park-Braswell, Sandra J. Shultz, and Randy J. Schmitz
}

\begin{abstract}
Context: Greater anterior knee laxity (AKL) is associated with impaired sensory input and decreased functional knee stability. As functional magnetic resonance imaging (MRI) is the gold standard for understanding brain function, methods to load the anterior cruciate ligament in the MRI environment could further our understanding of the ligament's sensory role in knee joint stability. Objective: To design and validate an MRI-compatible anterior knee joint loading device. Design: Descriptive laboratory study. Setting: University laboratory study. Participants: Sixteen healthy and physically active females participated (age = 23.4 [3.7] y; mass $=64.4[8.4] \mathrm{kg}$ ). Interventions: The AKL was assessed by a commercially available arthrometer. The AKL was also assessed with a custom-made, MRI-compatible device that produced anterior knee joint loading in a manner similar to the commercial arthrometer while obtaining dynamic structural MRI data. Main Outcome Measurements: The AKL (in millimeters) at $133 \mathrm{~N}$ of loading was assessed with the commercial knee arthrometer. Anterior displacement of the tibia relative to the femur obtained at $133 \mathrm{~N}$ of loading was measured from dynamic MRI data obtained during usage of the custom device. Pearson correlations were used to examine relationships between the 2 measures. The $95 \%$ limits of agreement compared the absolute differences between the 2 devices. Results: There was a 3.2-mm systematic difference between AKL (6.3 [1.6] mm) and anterior tibial translation $(3.2[1.0] \mathrm{mm})$ measures. There was a significant positive correlation between values obtained from the commercial arthrometer and the MRI-compatible device values $(r=.553, P=.026)$. Conclusions: While systematic differences were observed, the MRI-compatible anterior knee joint loading device anteriorly translated the tibia relative to the femur in a similar manner to a commercial arthrometer design to stress the anterior cruciate ligament. Such a device may be beneficial in future functional magnetic resonance imaging study of anterior cruciate ligament mechanoreception.
\end{abstract}

Keywords: anterior knee joint loading, MRI-compatible device, magnetic resonance imaging, validation

Anterior cruciate ligament (ACL) injury is one of the most common traumatic knee injuries sustained during sporting activity. ${ }^{1}$ Among the multiple risk factors of ACL injury, greater anterior knee laxity (AKL) is known as a strong independent predictor of ACL injury. ${ }^{2}$ It is suggested that individuals with greater AKL have decreased joint stability during physical movement (functional stability) due to poor proprioception and resultant altered movement patterns. ${ }^{3}$ This diminished functional stability in individuals with greater knee laxity may result in increased risk factors of ACL injury.

During functional joint stabilization, the brain plays an essential role in integrating and processing the sensory information arising from the peripheral area to generate a motor strategy that allows for joint stability. ${ }^{4}$ The neuroplastic properties of the brain allow for structural and functional changes to the brain in response to altered sensation. This neuroplasticity allows for modification to the neuronal circuits in response to alternated sensory stimulation. ${ }^{5}$ The loss of sensory information as a result of tissue damage incurred during orthopedic injury, such as ACL injury, has been demonstrated to result in altered brain activation patterns during lower leg movement tasks. ${ }^{6}$ This may indicate possible neuroplasticity in response to sensory alteration from the joint.

It is understood that mechanoreceptors in the ACL respond to anterior knee joint loading. ${ }^{4}$ Neuroimaging techniques such as functional magnetic resonance imaging (fMRI) and electro-

Park-Braswell is with Indiana Wesleyan University, Marion, IN, USA. Shultz and Schmitz are with the University of North Carolina at Greensboro, Greensboro, NC, USA. Park-Braswell (anne.park-braswell@indwes.edu) is corresponding author. encephalography allow us to measure the human brain function noninvasively during experimental tasks, such as joint loading. Previous work has assessed electroencephalography during commercial arthrometer anterior laxity testing designed to strain the ACL and elicit the firing of ACL mechanoreceptors. ${ }^{7}$ While electroencephalography can assess brain activation during joint loading, it is limited by poor spatial resolution when compared with magnetic resonance imaging (MRI) measures. ${ }^{8}$

The fMRI approaches to understanding brain function are currently limited with regard to the type of task/equipment that can occur in the scanner environment. The fMRI tasks are generally limited to supine positioning in the magnetic resonance (MR) with a critical need to immobilize the heads still to minimize motion-related noise. Moreover, any type of ferromagnetic material is contraindicated in the MR environment. Thus, there are technical difficulties in performing tasks designed to load the ACL in the MR environment.

The ability to perform anterior knee loading in a manner designed to stress the ACL in the MR environment may help researchers better understand how an established risk factor of ACL injury (knee joint laxity) may impact brain function. This ability to load the ACL in the MR environment would assist future work in understanding processing of sensory signals arising from the knee joint and subsequently help to identify brain function associated with sensorimotor control associated with knee joint stabilization. Therefore, we designed and constructed an MRI-compatible anterior knee joint loading device to apply a load similar to commercially available knee arthrometers (Figure 1). Thus, the purpose of this study was to design and validate an MRI-compatible anterior knee joint loading device that translates the tibia relative to the 


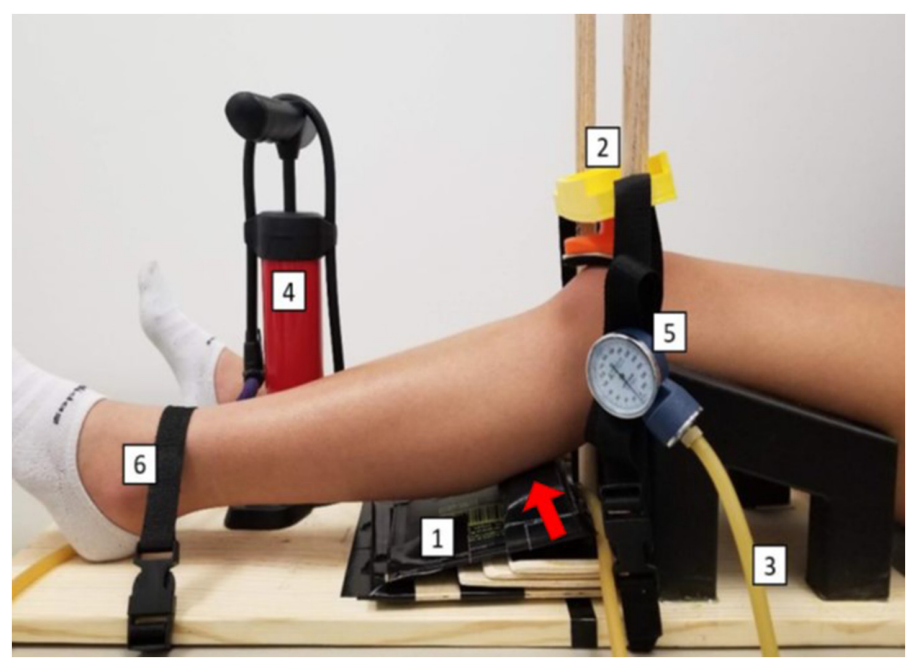

Figure 1 - MRI-compatible anterior joint loading device. (1) Air bladder, (2) patellar stabilizer, (3) latex tube, (4) bicycle pump, (5) airpressure gauge, and (6) ankle strap. The air bladder (1) located underneath the participant's calf will produce a force (arrow) to translate the tibia relative to the femur while stabilizing the patella (2) and ankle (6). The air bladder (1) is connected with latex tubing (3), and air pressure is provided by a manual pump (4) inflating the air bladder via latex tubing. The 189$\mathrm{mm} \mathrm{Hg}$ air pressure will be used to translate the tibia (equivalent to $133 \mathrm{~N}$ ), and it will be measured by air gauge (5). MR indicates magnetic resonance.

femur in the same manner as commercial arthrometers designed to assess anterior knee joint laxity.

\section{Method}

The MRI-compatible joint loading device was built to perform a passive anterior translation of the tibia. Tibial displacement measures obtained in the MRI environment were compared with commercial arthrometer measures. A total of 16 healthy and physically active female participants who were MR compatible volunteered for this study (age $=23.4$ [3.7] y; mass $=64.4$ [8.4] kg). First, AKL was measured using a KT-2000 knee arthrometer (Medmetric Corp, San Diego, CA). Participants were tested in the supine position with the knee flexed to $25^{\circ}\left(5^{\circ}\right)$. A Velcro strap was placed around their thigh to control hip external rotation. Then, the KT2000 was located on the participant's anterior aspect of the tibia, and the examiner applied $89 \mathrm{~N}$ of force in the posterior direction and $133 \mathrm{~N}$ of force in the anterior direction for 3 cycles. The investigator had previously established between-day measurement consistency and precision (intraclass correlation coefficient $=.97$; standard error of the mean $[\mathrm{SEM}]=0.5 \mathrm{~mm}$ ). The AKL was analyzed using the MATLAB software package (The MathWorks, Inc, Natick, MA).

Nonferromagnetic materials such as wood, plastic, and latex tubing were used to construct the MRI-compatible device (Figure 1). A patellar pad similar to the design of the KT-2000 restrained anterior femur motion. An anteriorly directed force was applied to the proximal tibia through a posteriorly positioned air bladder connected to a manual air pump. To ensure that a 133-N force was applied (force level common to commercial arthrometers), load validation outside of the MR environment was performed with the air bladder and an external dynamometer (Lafayette Instrument, Boston, MA). It was determined that $187 \mathrm{~mm} \mathrm{Hg}$ equated to $133 \mathrm{~N}$ of force by loading the air bladder at multiple inflation pressures while simultaneously assessing dynamometer load.

Then, dynamic MR knee images were obtained during knee joint loading using the device. Participants were positioned supinely inside the scanner with their leg in the MRI-compatible joint loading device (Figure 2). The air bladder was placed posterior to the participant's calf, and the patella/thigh was firmly stabilized. The air bladder was connected with a latex tube to the manual pump by passing through a small port in the wall between the MR scanning room and the adjacent operator room. Once the participant has been properly set up within the MRI-compatible joint loading device, a 36-channel large body coil (TIM System, Siemens Medical Solutions, Erlangen, Germany) was placed above the knee to obtain dynamic knee images (Figure 2). Anterior knee joint loading was performed from 0 to $133 \mathrm{~N}$ for a total of $5 \mathrm{load} /$ relaxation cycles over a duration of 36 seconds.

The MRI began with a localizer image that was used to prescribe the subsequent dynamic images of a single central sagittal slice of the knee joint. The dynamic MR acquisition parameters followed the methods of Quick et al, ${ }^{9}$ which obtained an image every $363 \mathrm{~ms}$ for 36.3 seconds, resulted in a total of 100 images (repetition time $=363 \mathrm{~ms}$; echo time $=160 \mathrm{~ms}$, and voxel size $=$ $1.1 \mathrm{~mm} \times 1.1 \mathrm{~mm} \times 4.0 \mathrm{~mm}^{9}$ ). It resulted in an effective $2.8-\mathrm{Hz}$ sampling frequency of a single sagittal slice.

Anterior tibial translation (ATT) was measured from the knee MR images using the open-source software MIPAV (version 8.0.2, Medical Image Processing, Analysis and Visualization; Center for Information Technology, National Institutes of Health, Bethesda, MD; https://mipav.cit.nih.gov). Specifically, the tibial tuberosity and the most anterior point of the femoral condyle were manually tracked by a single investigator through all 100 knee images (Figure 3A). The displacement from the unloaded joint to the peak maximum joint displacement was measured (Figure 3B). It was assumed that peak displacement occurred in conjunction with the peak physical load applied through the air bladder. Although every attempt was made to firmly stabilize the femur, a small amount anterior movement of the femur was still present at peak loading $(2.2[1.1] \mathrm{mm})$. Thus, we measured the ATT by subtracting the displacement of the femoral condyle from the displacement of tibial tuberosity. To establish intratester consistency and precision, the MRI-obtained ATT values for all the participants were measured twice by the same investigator (intraclass correlation coefficient $[3,1]=.95 ; \mathrm{SEM}=0.6 \mathrm{~mm}$ ). Pearson correlations were used to determine relationships between AKL and ATT values.

The statistical analyses were completed using the Statistical Package for Social Science (SPSS Inc, Chicago, IL). Pearson correlations were used to determine the relationship between AKL and ATT values. In addition, differences between AKL and ATT values were examined using $68 \%$ and $95 \%$ limits of agreement. The ATT value was subtracted from the AKL value, and $68 \%( \pm 1 \mathrm{SD})$ and $95 \%( \pm 1.96 \mathrm{SD})$ confidence intervals were constructed around the mean difference.

\section{Results}

The AKL value measured by the KT-2000 was 6.3 (1.6) mm, while ATT value measured by the MRI-compatible device was 3.2 (1.0) $\mathrm{mm}$. The AKL and ATT values were moderately correlated $(r=.553, P=.026)$. The spaghetti plot is shown in Figure 4. Table 1 lists the descriptive statistics and absolute differences between AKL and ATT values. The systemic difference was $3.2 \mathrm{~mm}$. In $68 \%$ of the cases, the true value of ATT produced by the joint 


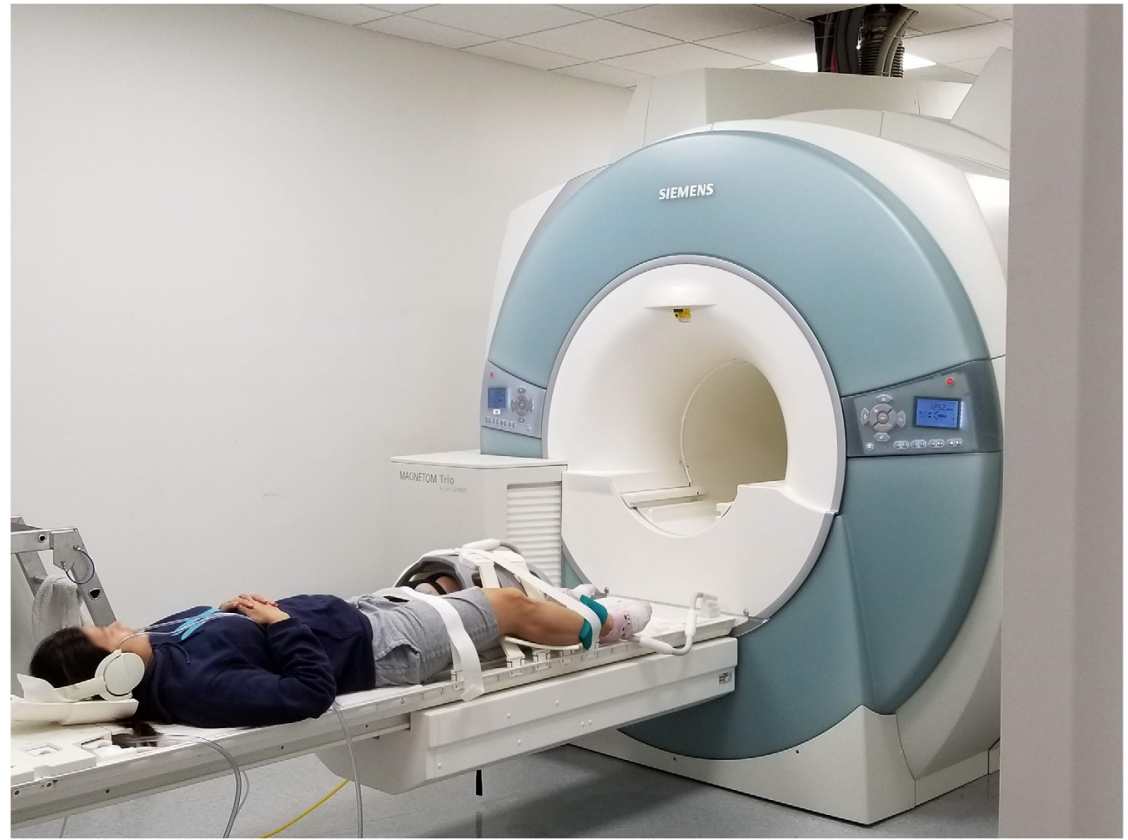

Figure 2 - Participant setup with magnetic resonance imaging-compatible anterior joint loading device.
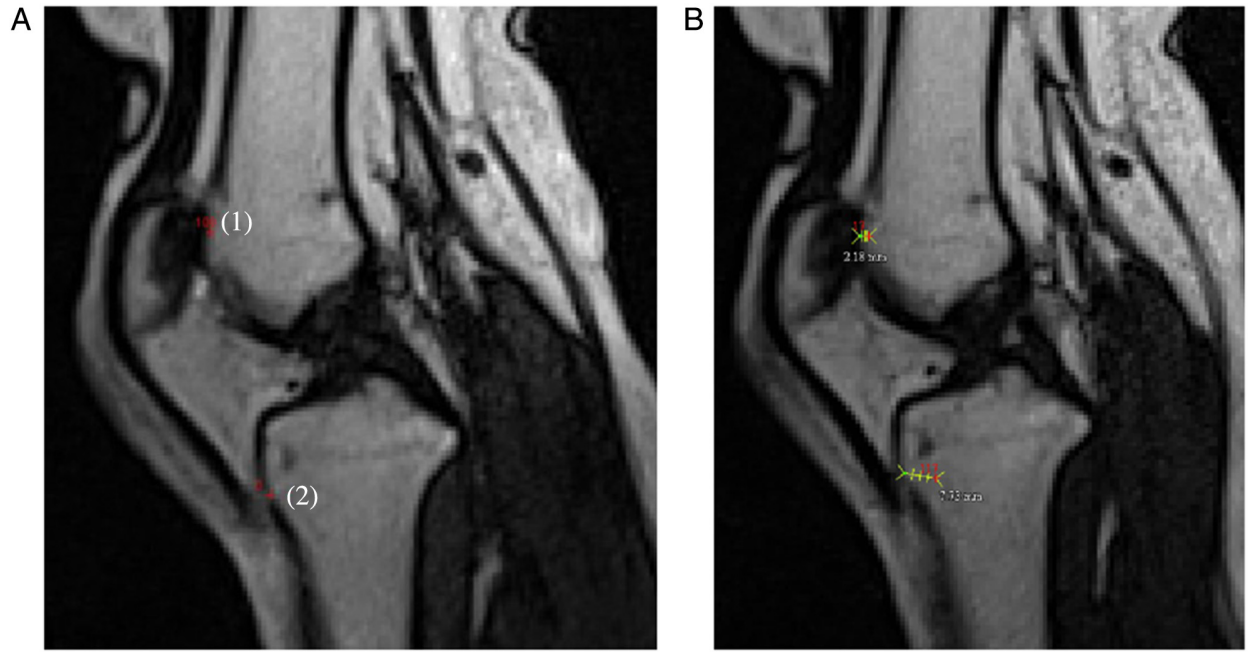

Figure 3 - Measuring ATT value using MIPAV. (A) The frame at zero joint load and (B) the frame with maximum anterior joint loading. (1) The most anterior point of the femoral condyle and (2) the point of the tibial tuberosity. ATT indicates anterior tibial translation; MIPAV, Medical Image Processing, Analysis and Visualization.

loading device as measured by MRI was within $1.4 \mathrm{~mm}$ of the AKL value measured by the KT-2000. In $95 \%$ of the cases, the true ATT value was within $2.7 \mathrm{~mm}$ of the AKL value.

\section{Discussion}

This study revealed that the MRI-compatible anterior knee joint loading device successfully translated the tibia relative to the femur. The ATT values were on average $3.2 \mathrm{~mm}$ less than AKL values measured with the KT-2000. These findings are consistent with Uh et al, ${ }^{10}$ who reported a $4 \mathrm{~mm}$ difference between KT measures and stress $\mathrm{X}$ rays when measuring total anterior-posterior knee laxity. These differences (both systematic and random differences) are likely due to the fact that MRI-derived ATT was a direct measure of tibiofemoral motion, while the KT2000-derived AKL approximated tibiofemoral motion based on relative displacement of pads on the tibial tuberosity and patella. Despite these differences, the 2 values were moderately correlated, suggesting that the MRI-compatible device loaded the tibia in a similar manner to the KT-2000 knee arthrometer.

Based on these findings, the MRI safe loading device described may be applicable for future fMRI studies designed to understand the central processing of knee joint mechanoreception under ligament loading. Understanding the neuronal activity during joint loading in individuals with high and low knee laxity may lead to a better understanding of the sensory role of the ACL and knee laxity as a risk for joint injury. Future studies are planned that 


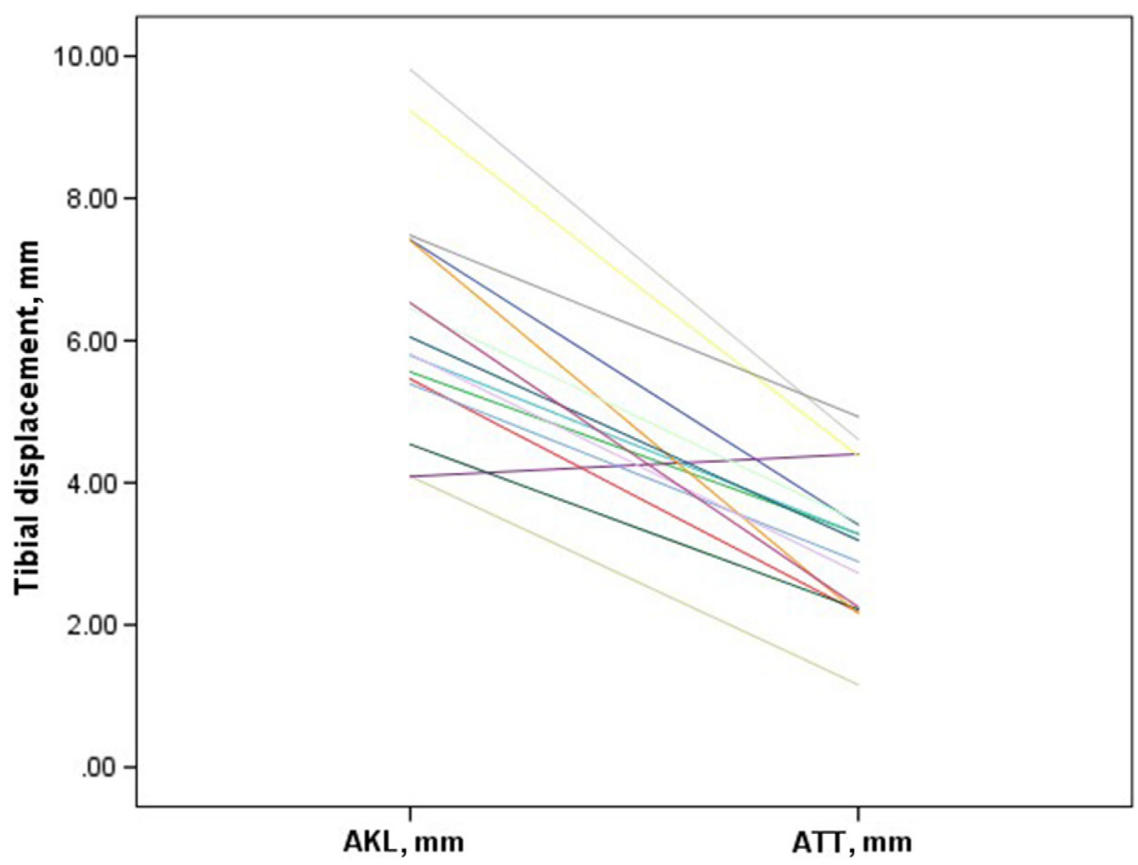

Figure 4 - Spaghetti plot of AKL and ATT data. AKL indicates anterior knee laxity; ATT, anterior tibial translation.

\section{Table 1 Mean (SD) for AKL and ATT Values, Mean Differences, and the $68 \%$ and $95 \%$ LOA for Mean Differences}

\begin{tabular}{|c|c|c|c|c|c|c|}
\hline \multicolumn{2}{|c|}{ AKL, mm } & \multicolumn{2}{|c|}{ ATT, mm } & \multirow[b]{2}{*}{$\begin{array}{c}\text { Mean } \\
\text { difference }\end{array}$} & \multirow[b]{2}{*}{$\begin{array}{l}68 \% \\
\text { LOA }\end{array}$} & \multirow[b]{2}{*}{$\begin{array}{l}95 \% \\
\text { LOA }\end{array}$} \\
\hline Mean & SD & Mean & SD & & & \\
\hline 6.3 & 1.6 & 3.2 & 1.04 & 3.2 & $3.2(1.4)$ & $3.2(2.7)$ \\
\hline
\end{tabular}

Abbreviations: AKL, anterior knee laxity; ATT, anterior tibial translation; LOA, limits of agreement.

will use the MRI-compatible joint loading device to identify the role of central processing and integrating components related to knee joint stabilization in individuals who are at high risk of ACL injury.

\section{Conclusion}

Our study measured the validity of the MRI-compatible anterior knee joint loading device using the KT-2000 knee arthrometer as the current clinical gold standard reference. Results demonstrated that the MR-compatible anterior knee joint loading device anteriorly translates the tibia in a manner similar to that obtained from a commercial arthrometer. However, the moderate absolute agreement between the measures suggests that it may be important to measure actual joint displacement while in the scanner to most accurately determine the loading stimulus to the ligament.

\section{References}

1. Prodromos CC, Han Y, Rogowski J, Joyce B, Shi K. A meta-analysis of the incidence of anterior cruciate ligament tears as a function of gender, sport, and a knee injury-reduction regimen. Arthrosc $J$ Arthrosc Relat Surg. 2007;23(12):1320-1325.e6. PubMed ID: 18063176 doi:10.1016/j.arthro.2007.07.003

2. Vacek PM, Slauterbeck JR, Tourville TW, et al. Multivariate analysis of the risk factors for first-time noncontact ACL injury in high school and college athletes: a prospective cohort study with a nested, matched case-control analysis. Am J Sports Med. 2016;44(6):1492-1501. PubMed ID: 27217522 doi:10.1177/0363546516634682

3. Rozzi S, Lephart S, Gear W, Fu F. Knee joint laxity and neuromuscular characteristics of male and female soccer and basketball players. Am J Sports Med. 1999;27(3):312-319. PubMed ID: 10352766 doi:10.1177/03635465990270030801

4. Kandel ER, Schwartz JH, Jessell TM. Principles of Neural Science. 4th ed. New York, NY: McGraw-Hill, Health Professions Division; 2000.

5. Johansson BB. Brain plasticity in health and disease. Keio J Med. 2004;53(4):231-246. PubMed ID: 15647628 doi:10.2302/kjm.53.231

6. Grooms DR, Page SJ, Nichols-Larsen DS, Chaudhari AMW, White SE, Onate JA. Neuroplasticity associated with anterior cruciate ligament reconstruction. J Orthop Sports Phys Ther. 2017;47(3):180-189. PubMed ID: 27817301 doi:10.2519/jospt.2017.7003

7. An YW, DiTrani Lobacz A, Lehmann T, et al. Neuroplastic changes in anterior cruciate ligament reconstruction patients from neuromechanical decoupling. Scand J Med Sci Sports. 2019;29(2):251-258. PubMed ID: 30326547 doi:10.1111/sms.13322

8. Scott H, Allen S, McCarthy G. Functional Magnetic Resonance Imaging. 3rd ed. Sunderland, MA: Sinauer; 2014.

9. Quick HH, Ladd ME, Hoevel M, et al. Real-time MRI of joint movement with trueFISP. J Magn Reson Imaging. 2002;15(6):710715. PubMed ID: 12112522 doi:10.1002/jmri.10120

10. Uh BS, Beynnon BD, Churchill DL, Haugh LD, Risberg MA, Fleming BC. A new device to measure knee laxity during weightbearing and non-weightbearing conditions. J Orthop Res. 2001;19(6):1185-1191. PubMed ID: 11781022 doi:10.1016/s0736-0266(01)00055-9 